\title{
Exploratory Identification of Image-Based Biomarkers for Solid Mass Pulmonary Tumors
}

\author{
Ifeoma Nwogu and Jason J. Corso \\ Department of Computer Science and Engineering, University at Buffalo, SUNY \\ 201 Bell Hall - Buffalo, New York 14260 \\ \{inwogu, jcorso\}@cse. buffalo.edu
}

\begin{abstract}
If imaging is to serve as a valid biomarker in the assessment of the response of cancer to therapies, a reproducible and predictive radiologic metric is required. A biomarker is an indicator of a biological property that can be used to measure the progress of disease. While current size-based, quantitative techniques provide numerical representations of tumors, they are not necessarily indicative of disease progression for advanced cancers. In this paper, we present an end-to-end process to explore the use of other image-based features especially statistical textural features for cancer change detection. We exploit the earth mover's distance metric for measuring the change in the tumor burden over a period, between the time the baseline scans were taken, and the time the therapy response scans were taken. The time-to-progression (TTP) of the disease is our known patient outcome. We analyze the correlations between TTP and our change measurements and discover that the local texture energy feature is most predictive of disease progression, more so than the tumor burden size on which current quantitative measures are made.
\end{abstract}

\section{Introduction}

Lung cancer is the leading cause of cancer deaths in North America and results in more deaths than breast, prostrate and colon cancer combined [1]. It is often diagnosed in late stages when surgery is no longer a viable treatment option and at this stage alternative therapies are required to manage the disease. As novel therapies become available, it is critical to rapidly assess their effectiveness for lung cancer treatment.

Imaging holds the promise of serving as an earlier, more accurate indicator of patient outcome than serologic or clinical parameters [2] [3] it can be noninvasive, is comparatively cheap, requires no extensive lab time, is nearly ubiquitous, and contains rich information describing the underlying cancer. But the large oncology groups and the Food and Drug Administration (FDA) require a reproducible radiologic metric to assess patient response to drug therapy if imaging is to serve as a biomarker [3] 4. A biomarker is an indicator of a specific biological property that can be used to measure the progress of disease or the effects of treatment.

D. Metaxas et al. (Eds.): MICCAI 2008, Part I, LNCS 5241, pp. 612 619, 2008.

(C) Springer-Verlag Berlin Heidelberg 2008 
The primary contribution of this study is to present an outcomes-driven process for evaluating image-based features that can potentially serve as imagebased biomarkers for quantifying and qualifying the extent of different cancers in the thoracic region. We also introduce the use of a robust method to compare tumor changes over time, and lastly, we show quantitatively that for advanced cancers, size might not be the optimal measure to assess disease progression.

The remainder of this section discusses the motivation and background of the study and section 2 discusses the materials used as well as the approach taken. Section 3 provides the results of our analysis as well as some observations and issues encountered during the study. Lastly, section 4 discusses our conclusions and directions for future work.

\subsection{Motivation}

CT is currently a very widely used imaging modality to assess the change in patient tumor burden. The tumor burden size is typically represented by basic quantitative values such as the two dimensional World Health Organization (WHO) [5] criterion or the Response Assessment Criteria for Solid Tumors (RECIST) one dimensional criterion 6]. From a dataset of 18 patients with advanced lung cancer, based on the RECIST measurements taken by four expert clinicians, 14 of the patients were classified as having stable disease with little or no disease progression over a given period of time. Of the 14, eight of the patients did not survive for more than twelve months after their progression date. One patient was recorded as having complete response, where the tumor burden has significantly reduced in size, but the patient only survived for about 3 months after the follow-up date.

These figures (though not statistically significant) present a snapshot of the current state of lung cancer tumor burden quantification - expert human readers currently using only size-based qualitative measurements such as RECIST, often give inaccurate prognosis for advanced cancers. We therefore investigate the correlations of additional features (texture and size) with the patient-outcome, time-to-progression (TTP), discussed in more detail in section 2.

\subsection{Background and Related Past Work}

A large percentage of published computer-aided diagnosis (CAD) research is focused on detecting lung cancer, where the main focus is the detection of lung nodules. Some successful nodule detection methods are given in [7, although this citation list is by no means exhaustive. In recent years, the FDA has approved several commercial CAD systems for nodule detection in the clinic and this research area appears stable today.

Typically, lung nodules are defined as pulmonary disorders less than $20 \mathrm{~mm}$ in diameter, and are always located in the lungs away from the hilar or root of the lungs, whereas solid mass lung tumors are about $200 \mathrm{~mm}$ or larger and can be located anywhere in the thoracic region. They can be buried in pleural effusion (fluid in the lungs), they can be located amidst blood vessels or as enlarged lymph 


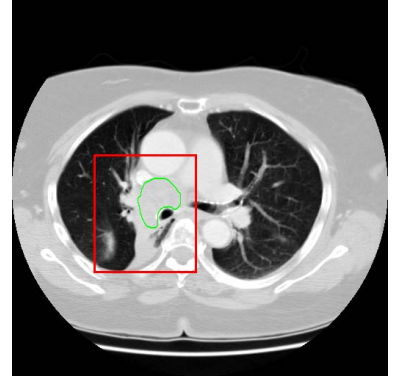

(a)

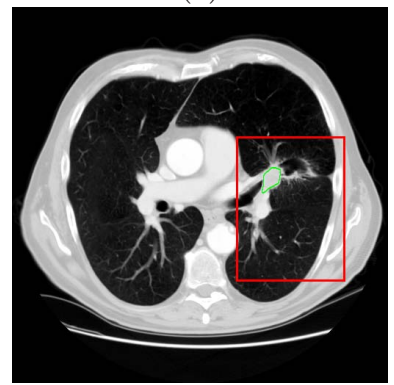

(d)

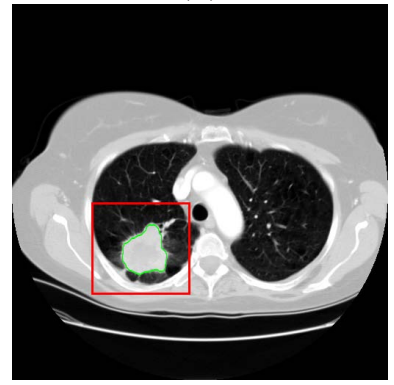

(g)

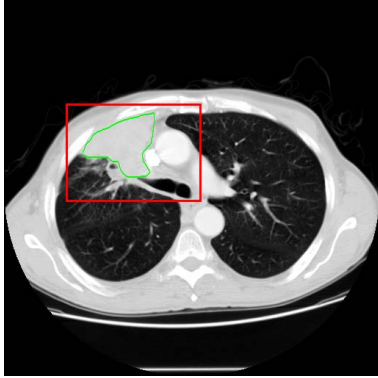

(b)

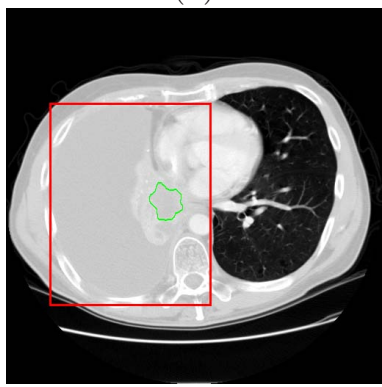

(e)

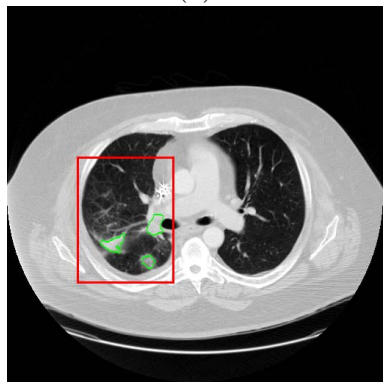

(h)

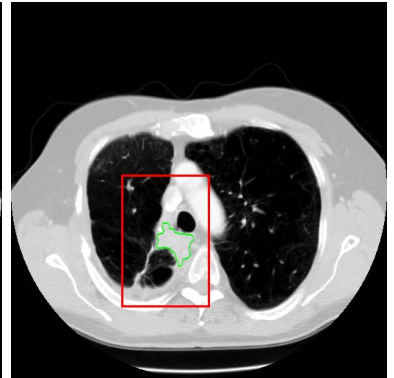

(c)

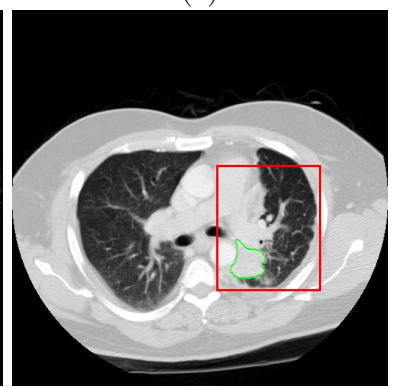

(f)

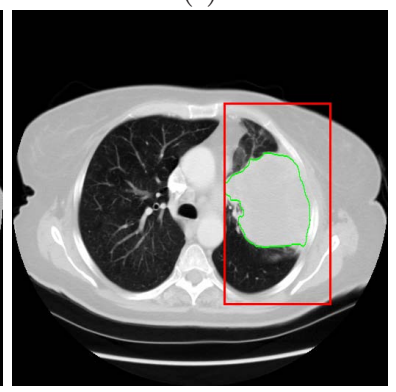

(i)

Fig. 1. CT slice images showing the ROI and contours of solid mass tumors in the thoracic region, highlighting the diversity in the tumor structures and locations

nodes, or in the mediastinum, etc. as shown in Figure 1, The most important characteristics for qualifying nodules appear to be size and growth rate, whereas there are no similar clear qualifiers for solid mass tumors. Also, lung nodules are structurally distinct in the thoracic region but solid mass tumors are very structurally diverse again as seen in Figure 1.

Hence, although CAD for early detection of lung cancer via lung nodules has been studied extensively, research in developing CAD techniques for solid mass chest tumors is still in its very early stages [7]. We are investigating the use of size and texture-based measurement techniques for quantifying lung cancer change detection. 


\section{Materials and Methods}

Our data set consists of two series of CT scans from eighteen patients with advanced lung cancer. One series of scans is the baseline taken before a particular therapy is administered to the patient, and the other series, the therapy response scans, are recorded after the patients is taken off the therapy. The response dates are recorded for each patient that survived beyond the duration of the treatment and this time period is recorded as the time-to-progression (in general, TTP is recorded when the disease is shown to change significantly). Also, the extent of survival for the patients is recorded. Hence, the known patient outcomes for this study are TTP and survival. Four expert radiologists also predicted the patient responses based on RECIST measurements. We restrict our analysis, in this study, to the primary tumor in each CT scan series.

Images are acquired using the 3-D CT scanner, GE Medical System LightSpeed QX/i helical, yielding 16-bit slices of $512 \times 512$ pixel arrays. The image values are recorded as Hounsfield Unit(HU) values, representing the densities of different human tissues. Because the tumors being investigated are very different in many of the cases (as shown in Figure 1), both manual and automatic processes are required to isolate the tumor regions-of-interest (ROI) and demarcate the tumor contours. The localization and demarcation results are then verified by our collaborating radiologists, to ensure the tumor regions and contours agreed with their findings. Computer-assisted localization and demarcation of solid mass lung tumor regions and contours is still an open area of research, essential for developing image-based biomarkers. We hypothesize that tracking density and vasculature changes in a tumor through its exhibited texture patterns, as well as tracking changes in the tumor burden size will provide deeper insight into detecting meaningful changes in the advanced cancer over time.

\subsection{Preprocessing}

Since the tissue densities we are currently interested in only extend over a fairly narrow range (or window), we shift the pixel values based on a window-level setting into the interval $[0,255]$. This shift is necessary not only for improved visualization, but to better perform the texture analysis. The mean density level of the window is set in such a way as to highlight tissue in the lungs as well as in the mediastinum and the width of the window affects the contrast of the resulting images. All CT slice images displayed in this paper result from the same window-level adjustments.

\section{$2.2 \quad$ Feature Extraction}

In most of the cases we evaluated, the tumor burden only occupied a small portion of the ROI. We therefore use a local texture analysis process based the construction of local gray-level co-occurrence matrices (GLCM). As mentioned in section 2.1. the CT slice image is down-scaled since the processing required to calculate a GLCM for the full dynamic range of a CT slice image is prohibitive. 

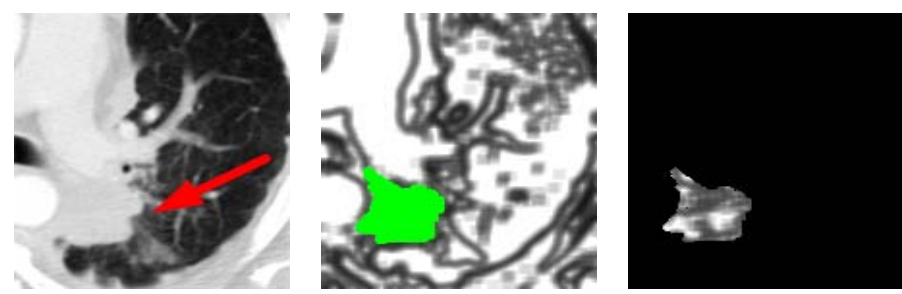

Fig. 2. Left: an example of an ROI indicating the tumor burden in 2D; center: the textured image (local energy) with the tumor mask applied; right: the input to the tumor signature

The statistical measurements we use are homogeneity, correlation, contrast and energy as defined by Haralicks's texture approaches [8], and implemented using the MATLAB Image Processing Toolbox.

Each ROI image is divided into overlapping $7 \mathrm{x} 7$ windows, the statistical texture values are computed on each window in four different directions and then averaged for rotational invariance. A mask of the specific tumor burden is then applied to the texture images resulting in texture representations of the tumor burden only. The tumor from each series therefore forms the input to the specific tumor signature. A signature is any variable length representation of a signal and is explained in more detail in section 2.3. Figure 2 shows how the texture image of a tumor is obtained. The size features are obtained as pixel count of the tumors within the demarcation contours. The tumor annotations were provided/validated by the expert clinicians, and they currently represent our best estimates of the tumor burden.

\subsection{Change Measurement Computation}

The Earth Mover's Distance is a metric function that measures the distance between two distributions 9]. EMD computes the minimal cost that must be paid in order to transform one distribution to the other. The EMD method is more robust than histogram matching techniques such as histogram intersection, the Kullback-Leibler divergence, Niblack quadratic-form distance and KolmogorovSmirnov distance, as shown by Rubner et al. in 9]. EMD operates on variable length representations (or signatures) and naturally allows for partial matching.

Our representative signature $\left\{\mathbf{s}_{j}=\left(\mathbf{m}_{j}, w_{\mathbf{m}_{j}}\right)\right\}$ is a set of feature clusters where each cluster is represented by its mean $\mathbf{m}_{j}$ and by $w_{\mathbf{m}_{j}}$, the fractional weight of that cluster. Our specific signature is made of histograms whose clusters are pre-determined by the binning size and whose weights are fractional values of the normalized histogram.

Given two signatures $P=\left(\mathbf{p}_{1}, w_{\mathbf{p}_{1}}\right), \ldots\left(\mathbf{p}_{m}, w_{\mathbf{p}_{m}}\right)$ with $m$ clusters and $Q=$ $\left(\mathbf{q}_{1}, w_{\mathbf{q}_{1}}\right), \ldots\left(\mathbf{q}_{m}, w_{\mathbf{q}_{m}}\right)$ with $n$ clusters; and $\mathbf{D}=\left[d_{i j}\right]$ where $d_{i j}$ the Euclidean distance between $\mathbf{p}_{i}$ and $\mathbf{q}_{j}$. We want to find a flow $\mathbf{F}=\left[f_{i j}\right]$ so that the cost 
equation 2 can be minimized subject to a given set of constraints given in detail in 9 .

$$
\operatorname{WORK}(P, Q, \boldsymbol{F})=\sum_{i=1}^{m} \sum_{j=1}^{n} d_{i j} f_{i j}
$$

so that

$$
\operatorname{EMD}(P, Q)=\frac{\sum_{i=1}^{m} \sum_{j=1}^{n} d_{i j} f_{i j}}{\sum_{i=1}^{m} \sum_{j=1}^{n} f_{i j}}
$$

Equation 2 defines the earth movers distance as the resulting work normalized by the total flow computed.

At the end of this process, we have eight signatures for each patient, four from the baseline scan and four from the therapy response scan. The four signatures represent the four local texture features. The EMD is then computed for each pair of texture feature signatures (one from baseline, one from therapy response) to obtain the measures of local texture change. To compute size changes, we take the absolute difference between the tumor burden size at baseline and at therapy response and divide by the value at baseline, yielding a fractional size change value.

\section{Experimental Results}

In this section we provide some numerical indicators and qualitative analysis to demonstrate the usefulness of the features investigated as potential candidates for image-based biomarker feature measures. We display five scatter plots in

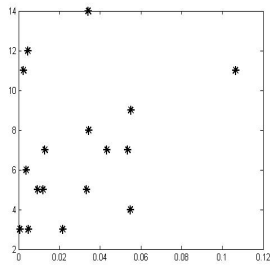

(a)

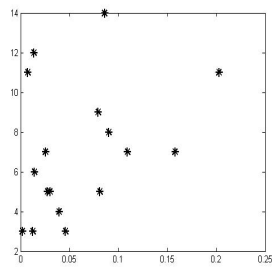

(d)

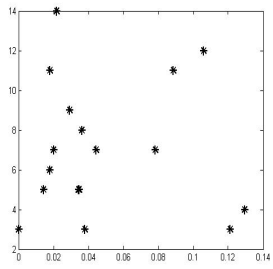

(b)

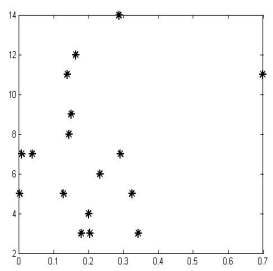

(e)

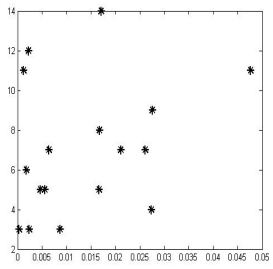

(c)

Fig. 3. (a)-(d) show the scatter plots of TTP (y-axis) versus the earth mover's distance between the texture features of a tumor taken at two different periods - local contrast, local texture correlations, local texture homogeneity and local energy (x-axis). (e) shows the scatter plot of TTP versus the fractional change in 2D tumor size. 
Table 1. The first four rows display the correlation coefficients for TTP values and the feature distance measures between the two readings obtained at two different time periods for each tumor. The last row shows the correlation coefficient for TTP and the fractional change in the tumor size, for readings obtained at two different time periods.

\begin{tabular}{|lccc|}
\hline Feature change measure & Low & Medium High \\
\hline EMD for local contrast & - & 0.3037 & - \\
EMD for local correlations & 0.0817 & - & - \\
EMD for local homogeneity & 0.2968 & - & - \\
EMD for local energy & - & 0.3356 & - \\
Fractional change in size & 0.1614 & - & - \\
\hline
\end{tabular}

Figure 3 to show the trend of the relationships between the measurements of feature changes (between baseline and therapy response times) and the patient outcomes, TTP. We also compute the correlation coefficients between the patient outcomes, TTP, and the feature changes to discover the degree of the linear relationship between them, and the resulting values are provided in Table 1 .

\subsection{Observations}

From reviewing the qualitative results in Figure 3] there is clearly a positive trend between the changes in the texture measurements and disease progression, although the correlations are not very strong. As expected, our analysis show that size, the current measurement standard, might not be a very good indicator of disease progression when the cancer is in its advanced stages.

Based on our analysis, we also observed that the best feature for measuring change detection is the local energy measure, using the EMD metric, and all the other texture features perform better than the size change measurement. Lastly, we observed that the local contrast and local homogeneity exhibit very similar change patterns and we can further explore this relation in the future.

\subsection{Issues with the Process}

Can TTP be reliably measured for advanced cancer? Using TTP as the primary patient outcomes measurement is very subjective as TTP values are only recorded when the clinicians believe that there is clear indication of disease progression. Different clinicians record TTP based on different criteria such as an increase in sum of tumor sizes, increase in pleural effusion (fluid in the lungs), enlargement of surrounding lymph nodes (different from the primary tumor), increase in the number of lesions present. In addition, TTP requires very careful follow-up.

Registration and delineation of the tumor are done on $2 \mathrm{D}$ slices limiting the spatial relationship measures between the pixels in a tumor, hence the texture and size feature measurements are not as accurate as they would be in a $3 \mathrm{D}$ model. 
In order to develop fully reproducible and repeatable biomarkers, there is a need for a semi- (or fully-)automated solid mass tumor detection procedure, but this is not the subject of this stage of the study.

\section{Discussion}

We presented an end-to-end outcomes-driven process for evaluating image-based features that can potentially serve as CT image-based biomarkers. We also highlighted some of the challenges involved in CAD for solid mass tumors in the thoracic region, a problem that needs to be sufficiently addressed in the development computer-generated image-based biomarkers for cancer change detection. Lastly, we provided some numerical indicators and qualitative analysis to demonstrate the usefulness of features investigated as potential candidates for image-based biomarker feature measures.

We intend to address the issues highlighted in section 3.2 and in addition, perform an even more thorough study to evaluate the role of the local windowing function for the GLCM texture analysis as well the use of other distance metrics including EMD with other methods of clustering.

Acknowledgements. The authors gratefully acknowledge the support provided by Drs. Ronald Gottlieb and Alan Litwin from the Radiology department at Roswell Park Cancer Institute; Janhavi Athale for her assistance in labeling the CT images. Ifeoma Nwogu was funded by the NSF award DGE-0333417.

\section{References}

1. Jemal, A., Tiwari, R.C., Murray, T., Ghafoor, A., Samuels, A., Ward, E., Feuer, E.J., Thun, M.J.: Cancer statistics, 2004. Cancer J. Clin. 54(8), 8-29 (2004)

2. Smith, J.J., Sorensen, A.G., Thrall, J.H.: Biomarkers in imaging: Realizing radiology's future. Radiology 227, 633-638 (2003)

3. El-Deiry, W., Sigman, C., Kelloff, G.: Imaging and oncologic drug development. J. Clin. Oncol. 24(20), 3261-3273 (2006)

4. Johnson, J.R., Williams, G., Richard, P.: End points and United States Food and Drug Administration approval of oncology drugs. J. Clin. Oncol. 21, 1404-1411 (2003)

5. World Health Organization: WHO Handbook for reporting results of cancer treatment (1979)

6. Therasse, P., Arbuck, S.G., Eisenhauer, E.A., Wanders, J., Kaplan, R.S., Rubinstein, L., Verweij, J., Van Glabbeke, M., van Oosterom, A.T., Christian, M.C.: New guidelines to evaluate the response to treatment in solid tumors. J. Natl. Cancer Inst. 92, 205-216 (2000)

7. Sluimer, I., Schilham, A., Prokop, M., van Ginneken, B.: Computer analysis of computed tomography scans of the lung: A survey. IEEE Trans. Med. Img. 25(4), 385-405 (2006)

8. Haralick, R.M.: Statistical and Structural Approaches to Texture. IEEE 67(5), 786804 (1979)

9. Rubner, Y., Tomasi, C., Guibas, L.J.: The earth mover's distance as a metric for image retrieval. Int. J. Comp. Vis. 40(2), 99-121 (2000) 